\title{
2 Design of computer experiments applied to modeling of compliant 3 mechanisms for real-time control
}

$\begin{array}{ll}4 & \text { Diego A. Acosta } \cdot \text { David Restrepo } \\ 5 & \text { Sebastián Durango } \\ \text { - Oscar E. Ruiz }\end{array}$

(C) Springer-Verlag London Limited 2012

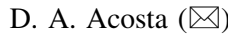

Calle 49 No 7-sur-50, Medellín, Colombia

e-mail: dacostam@eafit.edu.co

\section{Restrepo - O. E. Ruiz}

Calle 49 No 7-sur-50, Medellín, Colombia

\section{S. Durango}

Manizales. Antigua estación del Ferrocarril,
Received: 27 October 2010/ Accepted: 19 June 2012

\begin{abstract}
This article discusses the use of design of com-
\end{abstract} puter experiments (DOCE) (i.e., experiments run with a computer model to find how a set of inputs affects a set of outputs) to obtain a force-displacement meta-model (i.e., a mathematical equation that summarizes and aids in analyzing the input-output data of a DOCE) of compliant mechanisms (CMs). The procedure discussed produces a forcedisplacement meta-model, or closed analytic vector function, that aims to control CMs in real-time. In our work, the factorial and space-filling DOCE meta-model of CMs is supported by finite element analysis (FEA). The protocol discussed is used to model the HexFlex mechanism functioning under quasi-static conditions. The HexFlex is a parallel CM for nano-manipulation that allows six degrees of freedom $\left(x, y, z, \theta_{x}, \theta_{y}, \theta_{z}\right)$ of its moving platform. In the multi-linear model fit of the HexFlex, the products or interactions proved to be negligible, yielding a linear model (i.e., linear in the inputs) for the operating range. The accuracy of the meta-model was calculated by conducting a set of computer experiments with random uniform distribution of the input forces. Three error criteria were recorded comparing the meta-model prediction with respect to the results of the FEA experiments by determining: (1) maximum of the

Grupo de Investigación DDP, Universidad EAFIT,

Laboratorio de CAD CAM CAE, Universidad EAFIT,

Grupo de Investigación DM-DI, Universidad Autónoma de

Manizales, Colombia absolute value of the error, (2) relative error, and (3) root mean square error. The maximum errors of our model are lower than high-precision manufacturing tolerances and are also lower than those reported by other researchers who have tried to fit meta-models to the HexFlex mechanism.

Keywords Design of computer experiments - Design of experiments · Compliant mechanism - Meta-modeling . Plackett-burman design · Uniform design

\section{List of symbols}

XYZ Fixed reference coordinate system 41

T1 Input force port on Tab1 42

T2 Input force port on Tab2 43

T3 Input force port on Tab3 44

D1 Direction parallel to the connection beams in 45

D2 Direction perpendicular to the plane that contains 46

$\begin{array}{lll}\tau & \text { the HexFlex on its relaxed configuration } & \\ & \text { Vector of input forces and torques } & 47\end{array}$

r Configuration of the end effector $\quad 48$

$x, y, z \quad$ Coordinates of a point in XYZ frame $\quad 49$

$\theta_{x}, \theta_{y}, \theta_{z} \quad$ Set of $X Y Z$ Euler angles $\quad 50$

DOE Design of experiments 51

DOCE Design of computer experiments 52

CM Compliant mechanism 53

DOF Degrees of freedom 54

FEA Finite element analysis 55

PRBM Pseudo-rigid body modeling 56

\section{Introduction}

In traditional mechanisms, movement is achieved using kinematic joints (cylindrical, spherical, prismatic, etc.) and

5

6

47

48

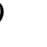

1

(

(

4

5

57


links that are as rigid as possible. In contrast, compliant mechanisms (CMs, [1]) are mechanical devices which undergo elastic deformations to transmit motion, force or energy from specified input ports to output ports. The main advantage of $\mathrm{CMs}$ with respect to traditional rigid-link mechanisms is that fewer parts and assembly processes, and no lubrication is required.

Due to the complexity of their motion (which is actually a deformation), CMs cannot be designed and directly analyzed by traditional kinematic methods [2]. Computational methods which require fast appraisal of the $\mathrm{CM}$ response to forces or, conversely, forces needed to bring the $\mathrm{CM}$ to a given configuration are needed for real-time control of the CM. Those methods usually imply the availability of a closed analytical (input/output, I/O) function, which can be computed directly and inversely. As a result of our work, the closed-form analytical function was found and, in addition, it is invertible around the operating point.

Two methods are used to relate CM deformations against forces and/or torques [3]: (1) pseudo-rigid body modeling (PRBM) and (2) numerical methods such as shooting methods (SM), finite element methods (FEM) and the chain algorithm [4]. In PRBM, the rigid body analysis is extended to the flexible-body analysis by finding the deflection of a flexible link by approximating closed-form functions $[4,5]$. This aspect considerably limits the applicability of PRBM as the approximated model is usually not accurate enough for precision applications [5]. On the other hand, if a numerical method is used to model CMs, the designer is basically solving a continuum mechanics problem by a discrete (computational) strategy. Therefore, the accuracy of the results critically depends on the resolution of the discretization. Since each run of the numerical solution requires considerable numerical processing, these methods are not suitable for time-critical applications (e.g., real-time control).

The term meta-model refers to an approximated I/O function to fit the I/O data produced by computer simulations of a model. Meta-modeling has been used to model a variety of complex systems. Reference [6] presents the application of meta-modeling on helicopter tests. Application of meta-modeling for vehicle testing analysis is presented in [7]. More general examples of applications of meta-modeling appear in Ref. [8, 9].

This article presents a design of computer experiments (DOCE) methodology to generate meta-models of CMs that synthesize a force (input)-displacement (output) model of CMs working under quasi-static conditions. In general, the lack of tools to model and analyze CMs is recognized as an open research problem [5], and these tools are actually required for real-time control. This methodology presents the advantages of DOCE that can be used to fit meta-models of any type of CMs.
The structure of this article is as follows: Sect. 2 presents a literature review and contrasts the contributions, Sect. 3 presents the proposed methodology and its scope for force-displacement modeling of CMs under quasi-static conditions, the case study "HexFlex" CM is developed in Sect. 4, where the proposed methodology is applied to obtain a mathematical meta-model that relates the actuator forces at the input ports with the end effector configuration. The mechanism, input factors (input forces), and their levels are described in Sect. 4.1. Section 4.3 develops the Fractional DOCE to determine the main factors. Section 4.4 presents the space filling DOCE and the meta-modeling of the HexFlex CM by conducting FEA tests. Section 4.6 successfully validates the meta-model just obtained by running 1,000 FEA tests and comparing their results against the meta-model predictions. Sections 5 and 6 conclude the article.

\section{Literature review, modeling of compliant mechanisms}

\subsection{Meta-models}

The term meta-model in computer experiments represents a surrogate model based on the use of statistical techniques to yield mathematical equations that approximate the results rendered by computer algorithms such as FEA [10]. If the true nature of a computer analysis code is $\mathbf{u}=\mathbf{u}(\mathbf{v})$ with $\mathbf{v}$ being the input variables vector and $\mathbf{u}$ the output variables vector from the computer code, then a surrogate model (i.e., meta-model) of the computer analysis is $\hat{\mathbf{u}}=\mathbf{z}(\mathbf{v})$ with $\hat{\mathbf{u}}$ being an approximation of the output variables defined by a functional relation $\mathbf{z}(\mathbf{v})$ found statistically. This carries an approximation error or residual defined as $\boldsymbol{\epsilon}=\hat{\mathbf{u}}-\mathbf{u}$.

Meta-models have benefits in screening variables, reducing design costs and optimizing designs [11]. They are applied here to model the quasi-static behavior of the HexFlex mechanism. The HexFlex is a six degrees of freedom parallel CM with distributed compliance for nanomanipulation designed at the MIT by Martin L. Culpepper and Gordon Anderson [12, 13].

\subsection{Force-displacement modeling of compliant} mechanisms

Topology and geometry optimization methods applied to CMs allow to tune up shapes, dimensions and connectivities to achieve a good numerical value of a function which evaluates the efficiency of the CM. The optimization relies on the possibility of relating forces/torques versus positions/deformations in the mechanism [3]. For such purpose,
159

160

161

$\begin{array}{lll}\text { Journal : Large } 366 & \text { Dispatch : 30-6-2012 } & \text { Pages : } \mathbf{1 5} \\ \text { Article No. : } \mathbf{2 6 8} & \square \text { LE } & \square \text { TYPESET } \\ \text { MS Code : } \quad \text { EWCO-D-10-00078 } & \sim_{\text {CP }} & { }_{\text {DISK }}\end{array}$


structural optimization uses either PRBM or numerically solved differential equations (FEA, SM, chain algorithms) or a conceptual synthesis tool [14] in which a building block approach is used to obtain a feasible initial design of known size and geometry and fine tuned using optimization methods. This literature review is focused on the analytical and numerical methods used as modeling tools in the analysis and design of CMs. A comparison of accuracy, computational efficiency and usability of methods for large deflection analysis of a cantilever beam (a specific CM member category) under free-end point load cases has been reported in Ref. [15], whose discussion and conclusions about the accuracy and computational efficiency of the studied methods are agree with our survey.

\subsection{Pseudo-rigid body modeling}

Pseudo-rigid body modeling is used to design CMs by representating compliant members by rigid link equivalents paired with standard joints (prismatic and revolute) and coupled with springs of appropriate stiffness [3, 14]. In this way, the CM behaves as a mechanism (i.e., has degrees of freedom) although strictly speaking no kinematic joints are present.

In the designed device, the PRBM differentiates stiff from flexible components. The first ones are modeled as completely rigid while the latter ones provide mobility to the mechanism (links coupled with displacement and torsional springs, non-linear elastic beams, etc.). These hyperflexible members can be analyzed with closed differential equations (e.g., flexural cantilever beam). A key step of the PRBM is to estimate the equivalent application point and equivalent elastic constant of the springs representing the compliant elements, i.e., the topology, geometry and elastic characteristics of the equivalent mechanism.

The PRBM approach is mathematically addressed under linear and non-linear strain formulations. This means that the strains are expressed in terms of linear and non-linear displacements. From elasticity theory, strains can be formulated as functions of the partial derivatives of the displacement functions, and, usually, higher-order partial derivatives.

The linear formulation neglects partial derivatives that have an order or power larger than one. The following articles present linear PRBM as a fundamental part of their formulation: analytical models of revolute and translational compliant joints are presented in Ref. [16]. In Ref. [17], PRBM is applied in predicting the behavior of a nano-scale parallel guiding mechanism which uses two carbon nanotubes as flexural links. The kinematic behavior was reported to be $92.7 \%$ accurate with respect to a molecular simulation. In Ref. [18], the kinematic and force analysis of compliant-driven robotic mechanisms is based on equations that relate joint torques, joint angles and displacements. In Ref. [19], the I/O model of a compliant micro-motion stage equivalent to a parallel mechanism formed by three limbs with rotational-rotational-rotational (3RRR) topology is obtained replacing the flexures with equivalent springs.

Non-linear PRBM is based mainly on the application of Euler beam models or deflection models based on the Castigliano's second theorem to model the flexible members of the CM solving high order partial derivatives of the strain formulation. The following articles present non-linear PRBM as part of their formulation: Ref. [20] discusses conic section flexure hinges using Euler beam model and Castigliano's second theorem. Reference [21] introduces an analytical approach to corner filleted flexure hinges using the Castigliano's second theorem. Reference [22] develops a synthesis and analysis PRBM for the limit configurations of a four-bar mechanism with an output compliant link (one end pinned to the coupler, one end fixed to the ground). The lumped compliance is modeled by non-linear beam theory, allowing for large non-linear deflections of the pinned end of the compliant link. The model only applies for a given topology. In Ref. [5], PRBM is enhanced to allow large deflections of elastic hinges. Four elastic hinges (leaf spring, cross, notch, and Haberland) are modeled and a joint-based modular approach is obtained. The modeling technique reported reduces the time needed for off-line modeling and design but not enough for real-time control. Reference [23] presents the mathematical model derived from the second Castigliano's theorem, for a six degrees of freedom (DOF) CM. The forward and inverse analyses of an open loop CM are developed in Ref. [24] using numerical methods to solve large deformations of the mechanism. Reference [25] develops a mathematical dynamic model, based on largedeflection beam models, for compliant constant force compression mechanisms. In Ref. [26], a large deflection analysis of compliant beams is presented. The method is based on the Adomian decomposition method in which differential equations are solved by a semi-analytical strategy different from the Euler beam or Castigliano's second theorem formulations. The method is reported to be efficient and accurate with respect to numerical and linear solutions. However, it is exclusively formulated for cantilever-like compliant members.

A model obtained with linear PRBM can be usually applied in real-time control but is restricted in precision engineering applications because of its low accuracy [1, 5]. Non-linear PRBM is suitable for accurate modeling and design, but it is not computationally efficient for real-time control. At any rate, PRBM requires that the geometry and loads of the elastic links allow for a closed-form analytic solution. These considerations seriously hinder the application of PRBM.

\begin{tabular}{|l|lll|}
\hline Journal : Large 366 & Dispatch : 30-6-2012 & Pages : 15 \\
Article No. : 268 & $\square$ LE & $\square$ TYPESET \\
MS Code : EWCO-D-10-00078 & $\sim_{\text {CP }}$ & $\checkmark$ DISK \\
\hline
\end{tabular}




\subsection{Numerical methods}

When the CM do not accept closed analytical force/ deformation solutions, and computing time is not an issue, numerical methods are applicable for their analysis and simulation. Reference [27] presents a procedure for the optimal design of flexural hinges for compliant micromechanisms. The optimal design is developed by coupling a FEA model to an optimization algorithm. The optimization is intended to maximize the rotation of the hinges under kinematic and strain constrains of the material of the hinge. Because of its time expenses, a pure FEA modeling of CMs is restricted to the design stage of the mechanism, being excluded from real-time control applications.

Reference [28] presents a localized application of FEA in CMs. In the design stage, two main steps are taken to complete a force-deformation model: (1) The elastic properties of the hinges are estimated by an independent 3D FEA. (2) The FEA-estimated properties are incorporated into a general CM model by the use of equivalent beams. This hybrid model may be applied in a reduced manner for real-time I/O models of CMs. The limitation exists because the geometry of the zones in which the equivalent flexible beam meets the rigid parts has a considerable influence on the predictions of deformations and stress concentrations of the CM.

Reference [29] presents the synthesis of CMs. The merit of the article is that, unlike others, it extensively presents the usage of highly non-linear finite elements, allowing the modeling of very large deformations.

In Ref. [30], the stiffness properties of a (compliant) notch hinge are computed using FEA relating the initial and final mechanism configurations under known loads. The procedure is only used to find the properties of the flexures and not to find an I/O model of the CM.

Reference [4] presents the use of a generalized shooting method (GSM) for the case of CMs with curved members. The method preserves the computational advantages of SM over finite differences and FEM: boundary value problems are treated as initial value problems instead of relying on fine discretization of the beam members to achieve high accuracy.

Summarizing, numerical methods such as FEM or SM are useful in determining the deflection and stresses in CMs because they allow to analyze CMs that have a geometry that is not easily modeled using methods like the PRBM. However, numerical methods cannot be used in a real-time scenario to control CMs. For this purpose, an intermediate I/O model must be estimated. This is the purpose of this investigation.

\subsection{Contribution of this article}

This article presents a new general procedure for modeling CMs under quasi-static conditions by DOCE methodology.
The proposed approach allows the modeling of CMs that have lumped or distributed compliance with simple or complex geometry. The main advantages of the proposed approach with respect to traditional modeling methods (PRBM, FEM, SM, chain algorithms) are:

1. The methodology is general enough to cover both lumped and distributed CMs.

2. The obtained input-output model might be simple enough to be used in real-time control.

3. Real experimentation is replaced by computer simulations reducing costs in product development.

It is clear that DOCE does not replace design of experiments (DOE) (i.e., physically conducted experiments). However, the pre-fitting of the model using DOCE serves to identify and avoid ranges, interactions and limitations that would make the DOE extremely expensive. As an application of the methodology, the 6 DOF CM HexFlex is modeled by finding an accurate model with respect to FEA simulations.

The differences in application domains between PRBM, numerical methods and DOCE techniques should be remarked here: PRBM is only applicable to mechanisms whose geometry can be decomposed into links for which an closed analytical expression for force versus deformations is possible (e.g. uniformly extruded beams). Numerical methods as FEM present no restrictions in the geometry of the mechanisms or bodies being analyzed. However, it is a slow method definitely not suited for realtime applications. DOCE allows to calibrate systems (not only mechanisms) and to obtain an I/O model which is fast and accurate for the chosen operation point. In our case, DOCE uses FEA as a subsidiary tool to carry the computer experiments and therefore allows to tune up the I/O model. In DOCE, if the mechanism or the operating point is changed, a new DOCE model is required. In a typical application of DOCE for mechanisms, a given mechanism is calibrated or modeled via computer experiments. Next, it is installed in the host device and then controlled via the analytical I/O model previously obtained.

\section{Methodology for meta-modeling of compliant mechanisms}

\subsection{Design of computer experiments}

In mechanism and machine science defining the configuration of a mechanism is equivalent to determining the positions of all moving points, or the location of all bodies, or specifying all joint parameters, etc. In this sense, defining the spatial configuration of a body (as the end effector of a $\mathrm{CM}$ ) is equivalent to determining six 
parameters, e.g. the three coordinates of a point and a set of three Euler angles. In meta-modeling of $\mathrm{CMs}$, we are seeking a function $f$ that relates the input forces and torques $(\tau)$ with the configuration $(\mathbf{r})$ of the end effector:

$f: \tau \rightarrow \mathbf{r}$

$\tau=\left[\tau_{1} \tau_{2} \cdots \tau_{n}\right]^{\mathrm{T}}$

$\mathbf{r}=\left[r_{1} r_{2} \cdots r_{m}\right]^{\mathrm{T}}$

with $m \leq n$.

A redundant mechanism occurs when $m<n$, while a non-redundant one occurs when $m=n$. In our discussion, we assume that the addressed mechanisms are not redundant. We also recall that for an end effector taking an spatial configuration, we have $m=6$. The methodology presented in Fig. 1 is proposed to model CMs under quasistatic conditions using meta-models from computational experiments. This methodology is based on results obtained by numerical simulations of CMs using FEA and is summarized as follows:

1. Define the topology and geometry of the CM. The topology concerns the connectivity and joint types of the CM. The geometry addresses the initial configuration as well as the exact shapes and sizes of the CM.

2. Define the set of factor parameters. That is, the vector of input forces $(\tau)$.

3. Propose a meta-model of the CM. This model is usually a multi-input multi-output (MIMO) polynomial model, calculated in the vicinity of the operating point. In Ref. [31], Rao states that MIMO regression models are an extension of SISO ones, whenever they have the same experimental design. Ordinary least squares (OLS) can be used for each individual output in independent manner. This usage has as a precondition that the outputs be actually independent from each other, while dependent on the input variables.

4. Use a fractional factorial DOCE (e.g., Plackett-Burman) to screen variables. The main factors are obtained by computer experiments. Fractional factorial DOEs can be used to screen $n$ factors when the number of runs is a power of 2 (i.e., $4,8,16,32, \ldots$ ). The gaps between these numbers widen considerably as $n$ increases [37]. Plackett and Burman deviced a fractional factorial DOE in which the number of runs is a multiple of four for any number $n$ of factors (i.e., 12, $20,24,28, \ldots)[32]$.

5. Use a space filling DOCE such as uniform design [33] to fine-tune the mathematical model of the mechanism by computer experiments.

6. Construct the surrogate model of the kinematics of the CM.
7. Verify the accuracy of the meta-model using extra experiments [10].

The previous steps define the scope of our article. However, it must be kept in mind that actual experiments must be conducted to fit an industrially applicable metamodel. Our contribution permits to execute these experiments with a minimum overhead cost.

\subsection{Methodology scope}

The presented methodology for modeling CMs is limited to:

1. CMs that allow small displacements of its end effector.

2. Input forces and moments slowly varying in time (quasi-static conditions).

3. The model is restricted to the neighborhood of the operation point for which was calculated.

4. Although the proposed methodology is general for $\mathrm{CMs}$, the obtained force-displacement models are specific for each individual case.

In spite of these limitations, the proposed force-displacement modeling of CMs by DOCE is relevant for a wide range of applications because most $\mathrm{CMs}$ are designed for small displacements of its end effector under quasistatic conditions, specially in compliant parallel nanomanipulating mechanisms. Because of this, they can be modeled by a low order Taylor series polynomial.

It should be emphasized that DOCE methodology is suitable when a empirical input-output model is required for a phenomenon or system which are fundamentally

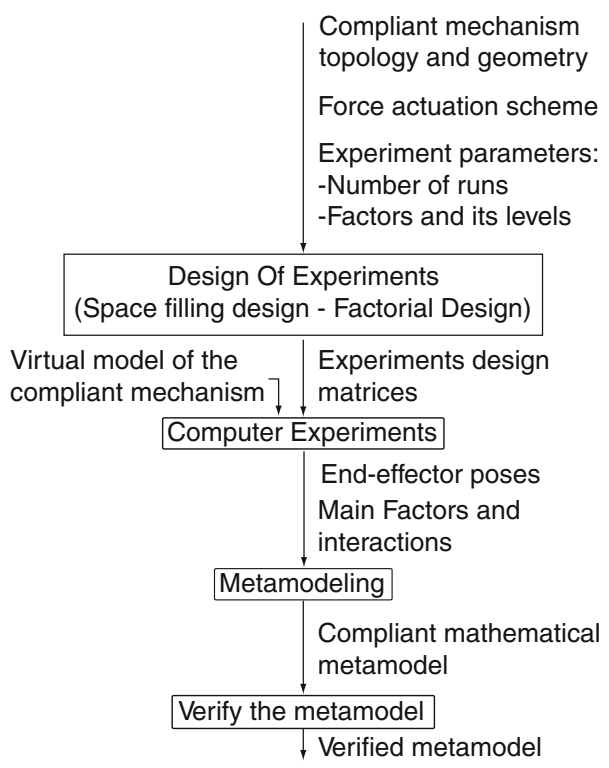

Fig. 1 Methodology for force-displacement meta-modeling of compliant mechanisms

$\begin{array}{lll}\text { Journal : Large 366 } & \text { Dispatch : 30-6-2012 } & \text { Pages : 15 } \\ \text { Article No. : } \mathbf{2 6 8} & \square \text { LE } & \square \text { TYPESET } \\ \text { MS Code : EWCO-D-10-00078 } & \sim_{\text {CP }} & \checkmark \text { DISK }\end{array}$


difficult to model theoretically. We fit a DOCE model which relates forces (inputs) to end effector configuration (outputs) of a mechanism. The application scenario of such a model is the real-time control of the mechanism in the vicinity of an operating point. Because one is interested in real-time control, an accurate but slow FEA model is out of question. The DOCE model might be a linear, quadratic, cubic, etc. approximation around the operating point. DOCE allows to trim the polynomial model by neglecting high level interactions if their statistical significance is low. In this article, this trimming led to a linear meta-model.

Section 4 shows the meta-modeling of the HexFlex CM under quasi-static conditions.

\section{Case study: force-displacement meta-modeling of the HexFlex mechanism}

Applying the procedure described in Sect. 3, the HexFlex parallel CM is meta-modeled.

\subsection{The HexFlex mechanism}

The topology and dimensions of the HexFlex are shown in Fig. 2. The HexFlex composed of a triangular moving platform, three tabs to provide an interface with the actuators, and six connection beams between the moving platform and the grounded zone (Fig. 2a). This mechanism allows the spatial motion of the moving platform, then, the end effector configuration is specified by six independent movements (e.g. translation and rotation, on the $X, Y$ and $Z$ axes) as shown in Fig. 3.

To control the moving platform, there are two actuators in the external edge of each tab. For each tab, one actuator acts in direction parallel to the connection beams (called direction one and denoted D1) and, the other actuator acts perpendicular to the tab ( $Z$ direction, D2) as in Fig. 4. Tabs are denoted T1, T2, T3. The motion of an specific actuator is denoted by the tab followed by the direction using the convention shown in Fig. 4.

The actuators used in the experiments allow a force of $1 \mathrm{~N}$. The positive direction of actuators for D2 coincides with the direction in which $Z$ is positive, and for D1 the positive direction of actuators is as shown in Fig. 4. Forces which vary slowly with time are assumed for the experiments (quasi-static experiments). Planar and non-planar displacements can be simultaneously achieved, by driving the tabs inside the planes $\Pi$ shown as an example in Tab 3 in Fig 3e. The material selected to model the mechanism is Aluminum 7075.

To define the meta-model function, the vector of input forces $(\tau)$ and end effector configuration $(\mathbf{r})$ are defined by:

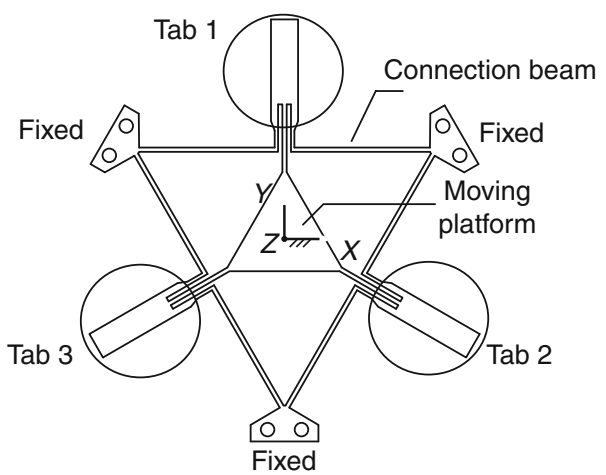

(a)

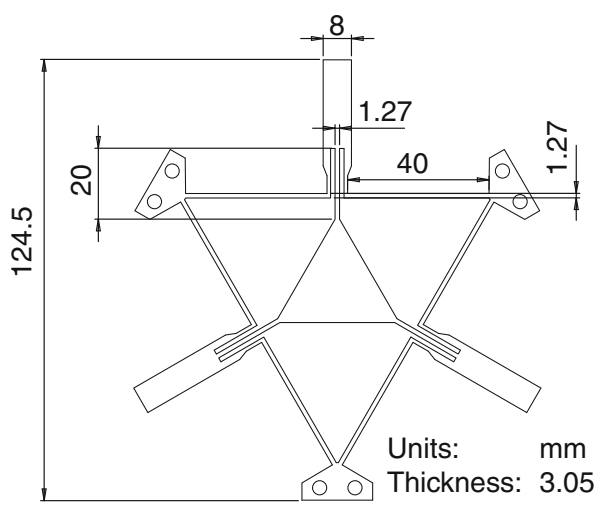

(b)

Fig. 2 Six DOFs compliant mechanism [34]. a HexFlex components. b HexFlex main dimensions

$$
\tau=\left[\begin{array}{llllll}
\mathrm{T} 1 \mathrm{D} 1 & \mathrm{~T} 1 \mathrm{D} 2 & \mathrm{~T} 2 \mathrm{D} 1 & \mathrm{~T} 2 \mathrm{D} 2 & \mathrm{~T} 3 \mathrm{D} 1 & \mathrm{~T} 3 \mathrm{D} 2
\end{array}\right]^{\mathrm{T}}
$$

$\mathbf{r}=\left[\begin{array}{llllll}x & y & z & \theta_{x} & \theta_{y} & \theta_{z}\end{array}\right]^{\mathrm{T}}$

where the end effector configuration is defined by six independent parameters: the coordinates $(x, y, z)$ of the moving platform center and a set $\left(\theta_{x}, \theta_{y}, \theta_{z}\right)$ of $X Y Z$ small Euler angles. Euler angles represent the final orientation of the moving stage as a succession of three rotations that take place around an axis whose location depends upon the preceding rotations [35]. However, in the case of small Euler angles, the order of the $X, Y$ and $Z$ rotations do not affect the represented orientation [36]. The input forces $\left(T_{i} D_{j}\right)$ correspond to the actuators in the tabs (tab $i$, force direction $j$ ).

The reference frame or world coordinate system is chosen to be at the center of the moving platform in its relaxed configuration (Fig. 3a). The moving platform coordinate frame is attached at its centroid. Therefore, in the relaxed configuration, the reference frame and the moving frame coincide.

\begin{tabular}{|l|lll|}
\hline & Journal : Large 366 & Dispatch : 30-6-2012 & Pages : 15 \\
Article No. : 268 & $\square$ LE & $\square$ \\
MS Code : EWCO-D-10-00078 & $\sim_{\mathrm{CP}}^{\text {TYPESET }}$ \\
\hline
\end{tabular}




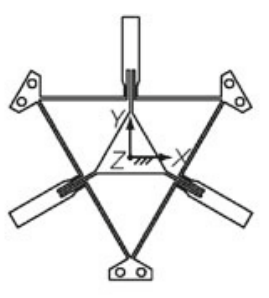

(a)

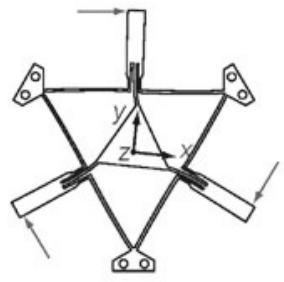

(c)

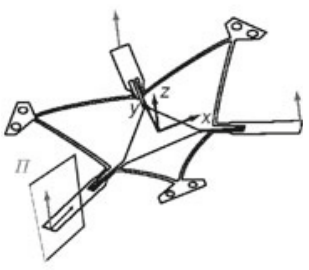

(e)

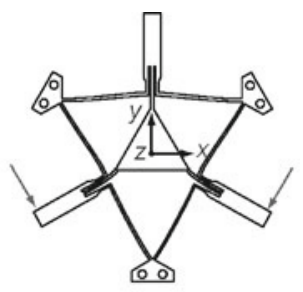

(b)

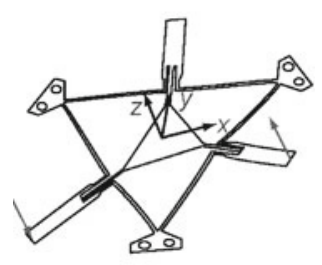

(d)

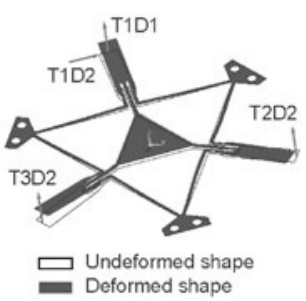

(f)

Fig. 3 Six DOFs compliant mechanism in deformation. a Relaxed position, $\mathbf{b}$ in-plane translations, $\mathbf{c}$ in-plane rotations, $\mathbf{d}$ out-plane rotations, e out-plane translations and $\mathbf{f}$ deformed shape

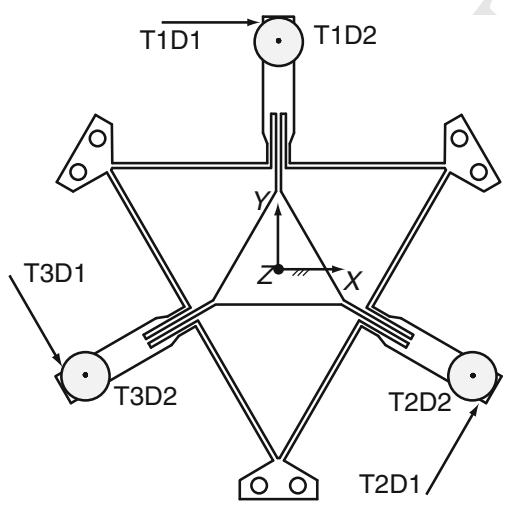

Fig. 4 HexFlex actuators direction

\subsection{Workflow of FE experiments and used software packages}

Figure 5 displays the assembly of computational tools to fit the DOCE model of CMs. This model is a set of hypersurfaces $\left(r_{1}, r_{2}\right.$, etc. $)$ in the multidimensional space of the input variables $\left(\tau_{1}, \tau_{2}\right.$, etc.), as shown in the upper right corner. The statistical fitting of the model (upper left corner of Fig. 5) is carried out with MATLAB ${ }^{\odot}$ code, using a Plackett-Burman DOE. Such values are calculated by a number of FEA simulations in ANSYS $^{\odot}$ (lower row of Fig. 5) controlled from the MATLAB $^{\odot}$ programs. ANSYS $^{\odot}$, therefore, acts in this case as a FEA server subordinated to MATLAB ${ }^{\odot}$ programs.

Using the symmetry of the mechanism and the dimensions shown on Fig. 2b, a sixth part of the mechanism was modeled and meshed to make a geometric FEM model of the mechanism (Fig. 6a). Using geometric transformations, the mechanism was completed developing a symmetrical mesh. Then the mesh was exported to ANSYS ${ }^{\odot}$ using quad shell elements to run the DOCE (Fig. 6b). The computer experiments obtained the moving frame configuration given a set of input loads on the tabs.

Operation ranges The selection of operation ranges for the DOCE is based on the recommendations of the HexFlex mechanism designers [34], corresponding to a force range of $\pm 1 \mathrm{~N}$. As for the operating point for the HexFlex mechanism, we adopted the usual one in the literature, which is $x=0, y=0, z=0, \theta_{x}=0, \theta_{y}=0$, and $\theta_{z}=0$. It must be pointed out that, in addition to the reviewed literature, the ranges, convenience of the operating point, and elimination of high degree and crossed-influence terms were verified by the series of computer experiments carried out by the methodology applied in this article.

The upper and lower levels of each factor for the fractional factorial and space filling DOCE are displayed on Table 1. The factors or inputs of the experiments are defined by Eq. (2) and correspond to the actuation forces of the mechanism, which are the controllable input variables (i.e., factors) of the experiment.

\subsection{Fractional factorial DOCE}

The statistical fitting of the model (upper left corner of Fig. 5) is carried out with MATLAB $^{\odot}$ code, using a

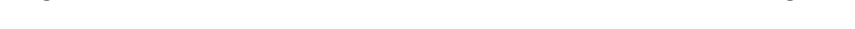

548

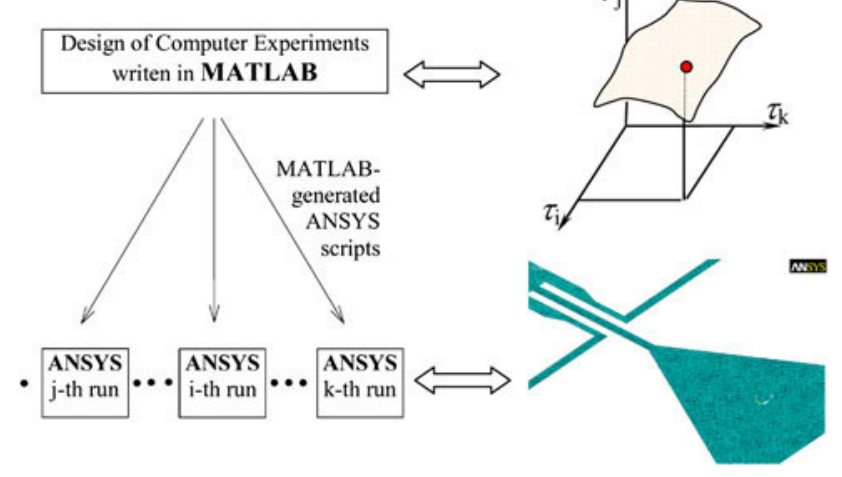

Fig. 5 ANSYS $^{\odot}$ as a FEA server controlled from MATLAB ${ }^{\odot}$

\begin{tabular}{|l|lll|}
\hline & Journal : Large 366 & Dispatch : 30-6-2012 & Pages : 15 \\
Article No. : $\mathbf{2 6 8}$ & $\square$ & $\square$ TYPESET \\
MS Code : EWCO-D-10-00078 & $\boldsymbol{V}_{\mathrm{CP}}$ & $\checkmark$ DISK \\
\hline
\end{tabular}




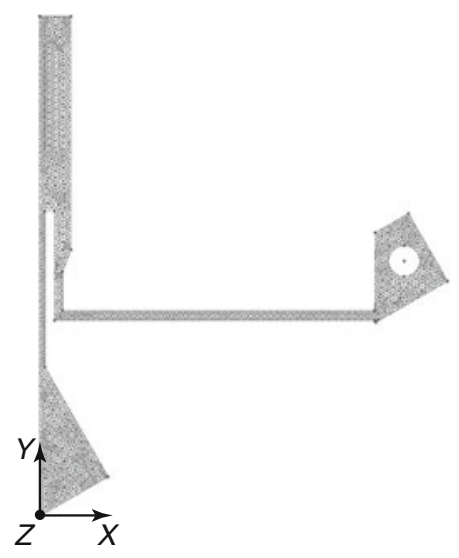

(a)

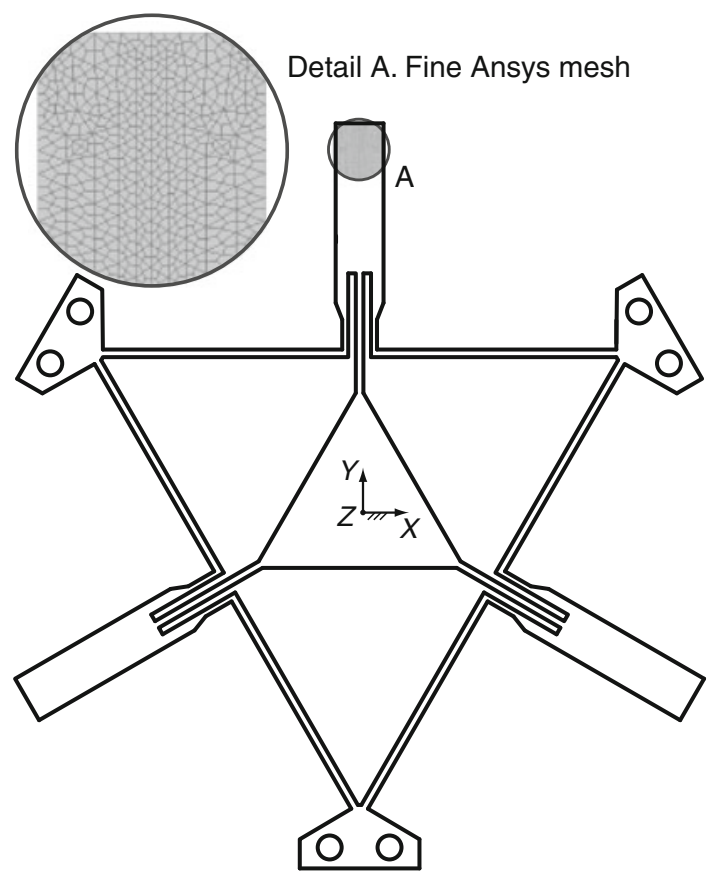

(b)

Fig. 6 FEA model of the HexFlex Mechanism. a Sixth part of the mesh. b FEM model in ANSYS ${ }^{\odot}$

fractional factorial DOCE. For this purpose, a PlackettBurman fractional factorial DOCE was chosen in lieu of a $2^{6-3}$ despite its higher number of runs (namely, 12) because of its higher resolution [37]. To screen factors, a Plackett-Burman (PB) DOCE [37-39] with 12 runs is made. Plackett-Burman designs are very economical and efficient when only main effects are of interest.

Provided that interactions are negligible (a hypotheis verified later using a uniform design of experiment), $\mathrm{PB}$ DOCE can be analysed using Lenth and Daniel plots [37]. Other accepted methods of analysis are the mean squared
Table 1 Studied factors

\begin{tabular}{lll}
\hline Factor & Low level $(\mathrm{N})$ & High level $(\mathrm{N})$ \\
\hline T1D1 & -1 & +1 \\
T1D2 & -1 & +1 \\
T2D1 & -1 & +1 \\
T2D2 & -1 & +1 \\
T3D1 & -1 & +1 \\
T3D2 & -1 & +1 \\
\hline
\end{tabular}

Forces in Tabs of the HexFlex

residuals and Bayes discrimination model determination [37]. In this paper, Lenth and Daniel plots are used to analyze PB DOCE and the assumption of negligible interactions is verified using a uniform DOCE. A script was developed to automatically generate the computer experiments and their results. The DOCE matrix and the results of each response are shown in Table 2.

To analyze the results of the PB DOCE, half normal probability (HNP) (Fig. 7) and Pareto (Fig. 8) plots are calculated. As an example, Fig. 8a means that $x \approx(11.5 \times$ $\mathrm{T} 1 \mathrm{D} 1+6 \times \mathrm{T} 2 \mathrm{D} 1+6 \times \mathrm{T} 3 \mathrm{D} 1) \times 10^{4}$. These analyses provide a simple way to examine the response variables (i.e., $x, y, z$, etc) and the relative importance of the factors of the experiment. The Pareto plots results coincide with half normal probability (HNP) showing that the main effects are consequent with the symmetries of the topology of the mechanism (Fig. 4). The symmetries of the mechanism also made that some effects had the same value.

In addition to the Pareto plots and HNP analysis, another way of looking at the resulting effects consists of using Lenth's plot [40]. The absolute values of the alias of the effects (i.e., estimate of an effect also including the influence of one or more other effects (usually high order interactions) in a fractional factorial DOE [38]) are ordered in ascending order to calculate the median $(v)$. Once the median is calculated a pseudo-standard error $\left(S_{0}\right)$ is estimated using the formula: $S_{0}=1.5 v$. The pseudo-standard error serves to define the margin of error (ME) and the simultaneous margin of error, using the 0.975-quantile and $t_{\mathrm{g}, \mathrm{m} / 3}$ of the $t$ student distribution allowing fractional degrees of freedom. The results for these analyses are displayed on Table 3 and indicate which independent variables (T1D1, T1D2, T2D2, T3D2, T2D1, T3D1) have effects on which dependent variables $\left(x, y, z, \theta_{x}, \theta_{y}, \theta_{z}\right)$. Because this is a fractional factorial design, we can only screen at this point the existence of such dependency. Later on, using a space filling technique we will confirm and quantify them. The responses are affected as follows:

1. $x$ and $\theta_{z}$ are mainly affected by $T 1 D 1, T 2 D 1$, and $T 3 D 1$.

\begin{tabular}{|l|lll|}
\hline & Journal : Large 366 & Dispatch : 30-6-2012 & Pages : 15 \\
Article No. : $\mathbf{2 6 8}$ & $\square_{\mathrm{LE}}$ & $\square$ \\
MS Code : EWCO-D-10-00078 & $\boldsymbol{V}_{\mathrm{CP}}^{\mathrm{TY}}$ & $\checkmark$ DISK \\
\hline
\end{tabular}


Table 2 Plackett-Burman DOCE matrix for 6 factors and 12 runs

\begin{tabular}{|c|c|c|c|c|c|c|c|c|c|c|c|}
\hline \multicolumn{6}{|c|}{ Design matrix } & \multicolumn{6}{|l|}{ Responses } \\
\hline $\begin{array}{l}\text { T1D1 } \\
\text { (N) }\end{array}$ & $\begin{array}{l}\text { T1D2 } \\
\text { (N) }\end{array}$ & $\begin{array}{l}\text { T2D1 } \\
(\mathrm{N})\end{array}$ & $\begin{array}{l}\text { T2D2 } \\
(\mathrm{N})\end{array}$ & $\begin{array}{l}\text { T3D1 } \\
(\mathrm{N})\end{array}$ & $\begin{array}{l}\text { T3D2 } \\
(\mathrm{N})\end{array}$ & $x(\mu \mathrm{m})$ & $y(\mu \mathrm{m})$ & $z(\mu \mathrm{m})$ & $\theta_{x}(\mu \mathrm{rad})$ & $\theta_{y}(\mu \mathrm{rad})$ & $\theta_{z}(\mu \mathrm{rad})$ \\
\hline 1 & -1 & 1 & -1 & -1 & -1 & 115,056 & 0.6 & $-862,976$ & 0.0001 & -0.0001 & 3.10176 \\
\hline-1 & -1 & 1 & 1 & 1 & -1 & $-57,529$ & $99,636.5$ & $-287,659$ & -39.3596 & -68.0656 & 3.10183 \\
\hline 1 & -1 & -1 & -1 & 1 & 1 & -3 & -0.6 & $-287,655$ & -39.2665 & 68.1194 & -9.30545 \\
\hline-1 & -1 & -1 & -1 & -1 & -1 & $-57,525$ & $-99,636.5$ & $-862,976$ & 0.0001 & -0.0001 & 3.10186 \\
\hline 1 & 1 & -1 & 1 & 1 & -1 & -3 & -0.6 & 287,655 & 39.2665 & -68.1194 & -9.30545 \\
\hline 1 & 1 & 1 & -1 & 1 & 1 & 57,525 & $99,636.5$ & 287,659 & 39.3596 & 68.0656 & -3.10186 \\
\hline-1 & -1 & -1 & 1 & 1 & 1 & $-115,056$ & -0.6 & 287,662 & -78.6262 & 0.0539 & -3.10176 \\
\hline-1 & 1 & -1 & -1 & -1 & 1 & $-57,525$ & $-99,636.5$ & 287,659 & 39.3596 & 68.0656 & 3.10186 \\
\hline-1 & 1 & 1 & 1 & -1 & 1 & 3 & 0.6 & 862,976 & -0.0001 & 0.0001 & 9.30545 \\
\hline-1 & 1 & 1 & -1 & 1 & -1 & $-57,529$ & $99,636.5$ & $-287,662$ & 78.6262 & -0.0539 & 3.10183 \\
\hline 1 & 1 & -1 & 1 & -1 & -1 & 57,529 & $-99,636.5$ & 287,655 & 39.2665 & -68.1194 & -3.10183 \\
\hline 1 & -1 & 1 & 1 & -1 & 1 & 115,056 & 0.6 & 287,662 & -78.6262 & 0.0539 & 3.10176 \\
\hline
\end{tabular}

2. $z$ and $\theta_{x}$ are mainly affected by $T 1 D 2, T 2 D 2$, and $T 3 D 2$.

3. $y$ is mainly affected by $T 2 D 1$ and $T 3 D 1$.

4. $\theta_{y}$ is mainly affected by $T 2 D 2$ and $T 3 D 2$.

Also, it is evident that to obtain in-plane displacements $\left(x, y, \theta_{z}\right)$, actuators should act in direction one (D1) and out-of-plane displacements $\left(\mathrm{z}, \theta_{x}, \theta_{y}\right)$ are generated when actuators act in direction two (D2).

\subsection{Space filling DOCE and meta-model of the HexFlex}

The preliminary assumption of negligible second-order effects was made in order to screen factors on the PlackettBurman fractional factorial design. Because such assumption had to be verified, a surface response design needs to be implemented to confirm the neglect of the second order effects assumed in the PB DOCE. Amongst all choices available, the uniform design was chosen because the physics of the problem at hand prevented using center points required by other response surface DOEs such as the central composite design. In other words, the center point would correspond to the relaxed configuration of the mechanism where no movement is achieved.

To generate a valid meta-model of the HexFlex, a uniform DOCE [41] was used with the same six factors shown in Table 1 and six evenly distributed levels (i.e., -1 , $-0.6,-0.2,0.2,0.6,1)$. A uniform design is a modification of fractional factorial designs that provides scatter design points in the experimental domain space. The design matrix and the FEA output displacements were calculated using ANSYS $^{\odot}$ (Table 4).
4.5 HexFlex mechanism meta-modeling

After running the space filling DOCE (Sect. 4.4), the next step consists of choosing an appropriate approximation model. Low-order polynomials have been used effectively for building approximations in a variety of applications including force-displacement modeling [42]. Here a second-order polynomial with interactions is used for metamodeling an input-output of the HexFlex.

The chosen polynomial model for the input-output meta-model of the HexFlex is shown on Eq. (4).

$r_{i}=\beta_{0}+\sum_{i=1}^{k} \beta_{i} \tau_{i}+\sum_{i=1}^{k} \beta_{i i} \tau_{i}^{2}+\sum_{i=1}^{k} \sum_{j=1}^{k} \beta_{i j} \tau_{i} \tau_{j}$

where $i=j=1, \ldots, 6, i<j, \tau_{i}$, and $r_{i}$ are components of $\tau$ and $\mathbf{r}$, respectively, as defined in Eqs. (2) and (3).

The Placket-Burman DOCE analysis points out which interactions of the behavior of the mechanism are not important and are therefore negligible in Eq. (4). Also, from preliminary experiments it is determined that the non-linear terms of Eq. (4) do not influence the end effector motion and are neglected. As consequence, the meta-model results in a system of six linear equations for the $x, y, z$, etc. motions. We obtained the force-displacement meta-model writing the system of linear equations as a matrix equation (Eq. 5).

$$
\begin{aligned}
& {\left[\begin{array}{lllllll}
x & y & z & \theta_{x} & \theta_{y} & \theta_{z}
\end{array}\right]^{\mathrm{T}}} \\
& =S_{\mathrm{T}}\left[\begin{array}{llllll}
\mathrm{T} 1 \mathrm{D} 1 & \mathrm{~T} 1 \mathrm{D} 2 & \mathrm{~T} 2 \mathrm{D} 1 & \mathrm{~T} 2 \mathrm{D} 2 & \mathrm{~T} 3 \mathrm{D} 1 & \mathrm{~T} 3 \mathrm{D} 2
\end{array}\right]^{\mathrm{T}}
\end{aligned}
$$

where $S_{\mathrm{T}}$ (Eq. 6) is the matrix representing the input-output first-order effects. Each term of $\mathrm{S}_{\mathrm{T}}$ is found using a least squares regression $[43,44]$. The units associated to the elements $S_{\mathrm{T}}$ for the HexFlex are $\mu \mathrm{m} / \mathrm{N}$ for rows $1-3$, and $\mu \mathrm{rad} / \mathrm{N}$ for rows $4-6$. 
$S_{\mathrm{T}}=\left[\begin{array}{cccccc}57.5 & 0 & 28.8 & 0 & -28.8 & 0 \\ 0 & 0 & 49.8 & 0 & 49.8 & 0 \\ 0 & 287.7 & 0 & 287.7 & 0 & 287.7 \\ 0 & 39,313.0 & 0 & -19,679.9 & 0 & -19,633.3 \\ 0 & 0 & 0 & -34,032.0 & 0 & 34,060.2 \\ -3,101.8 & 0 & 3,101.8 & 0 & -3,101.8 & 0\end{array}\right]$

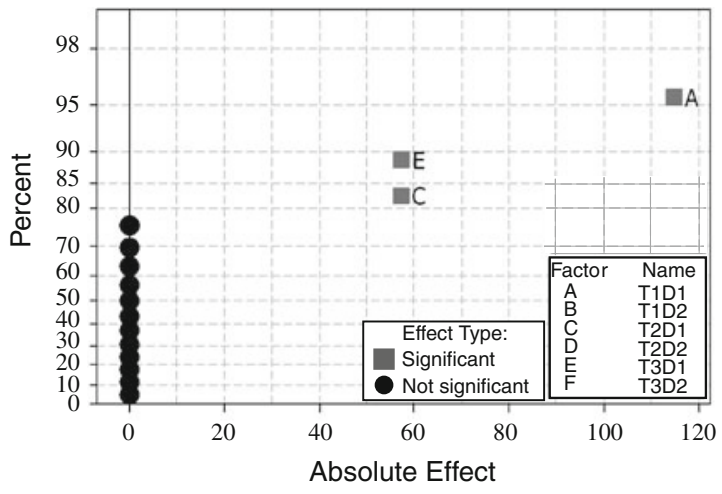

(a) Half Normal Plot for $x$ translation

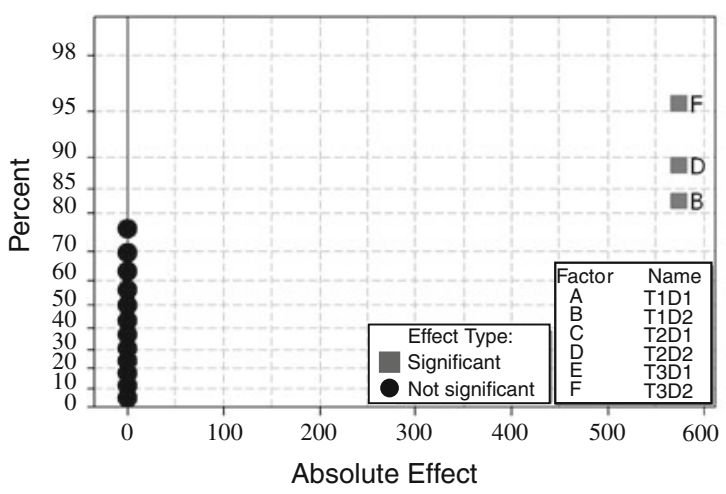

(c) Half Normal Plot for $z$ translation

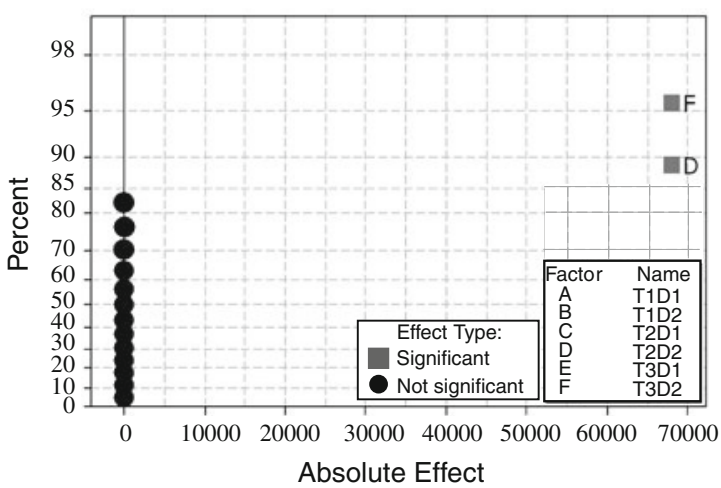

(e) Half Normal Plot for $\theta_{y}$ translation

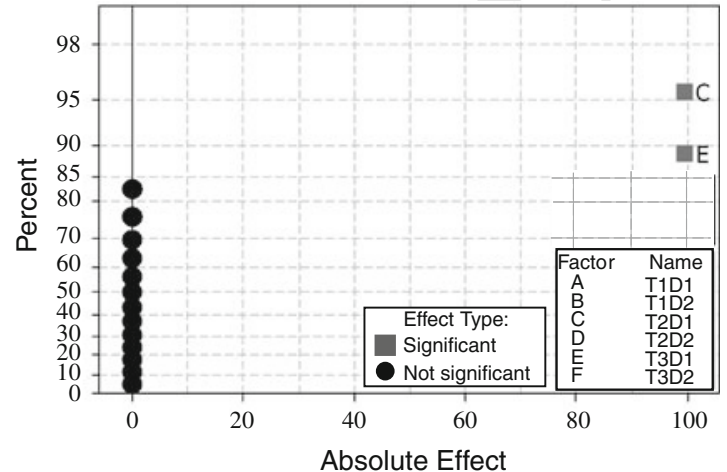

(b) Half Normal Plot for $y$ translation

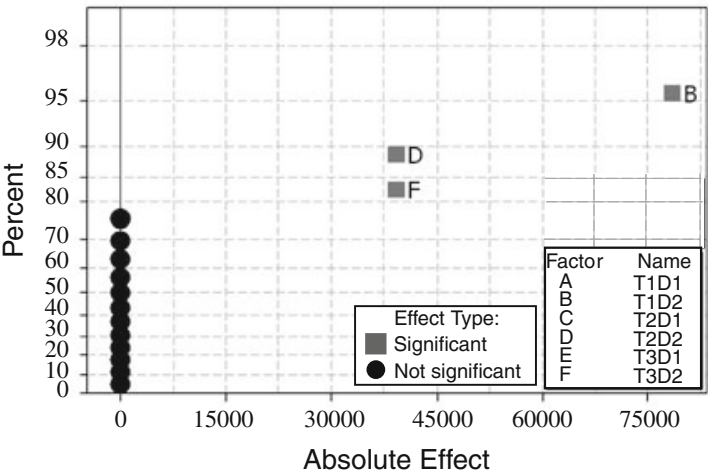

(d) Half Normal Plot for $\theta_{x}$ translation

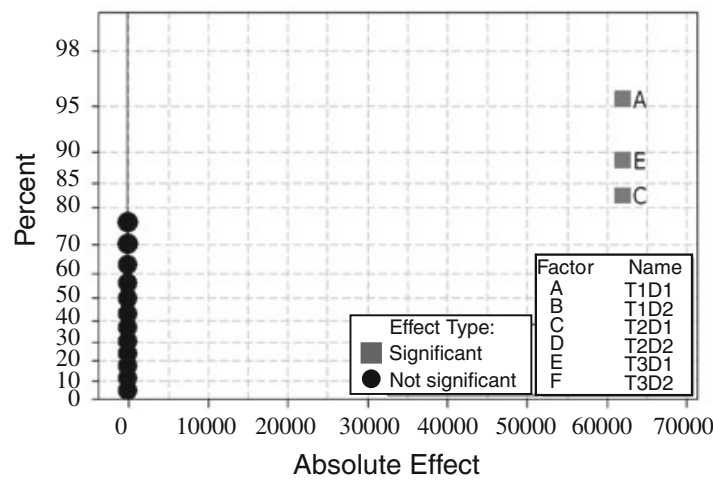

(f) Half Normal Plot for $\theta_{z}$ translation

Fig. 7 Half normal probability plots. Placket-Burman DOCE for 12 runs and 6 factors for HexFlex quasi-static conditions

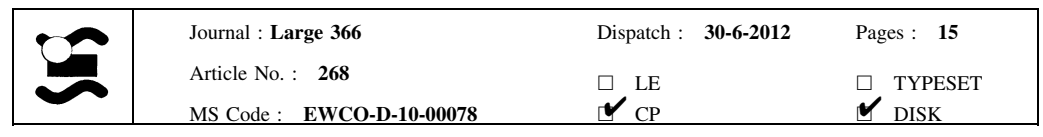


Fig. 8 Placket-Burman DOCE for 12 runs and 6 factors for HexFlex quasi-static conditions. a Pareto coefficients for $x$ translation $\left(\times 10^{4}\right)$. b Pareto coefficients for $y$ translation $\left(\times 10^{4}\right)$. c Pareto coefficients for $z$ translation $\left(\times 10^{5}\right)$. d Pareto coefficients for $\theta_{x}$ rotation. e Pareto coefficients for $\theta_{y}$ rotation. f Pareto coefficients for $\theta_{z}$ rotation

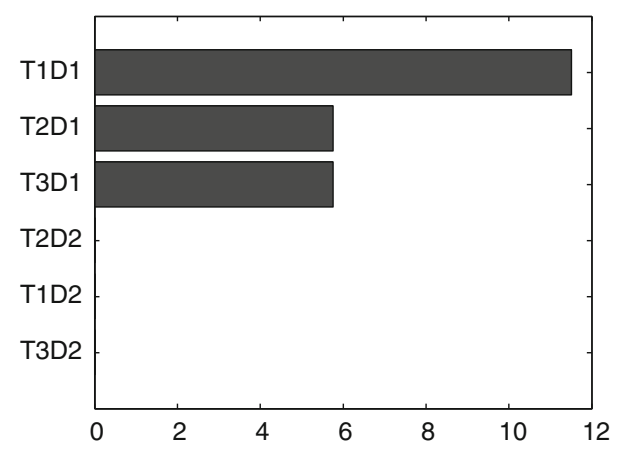

(a)

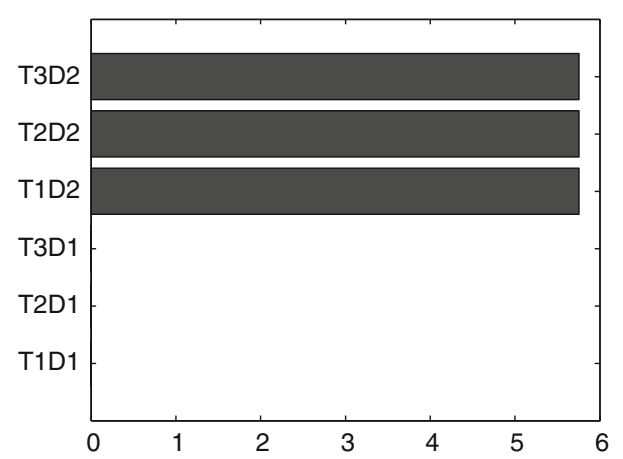

(c)

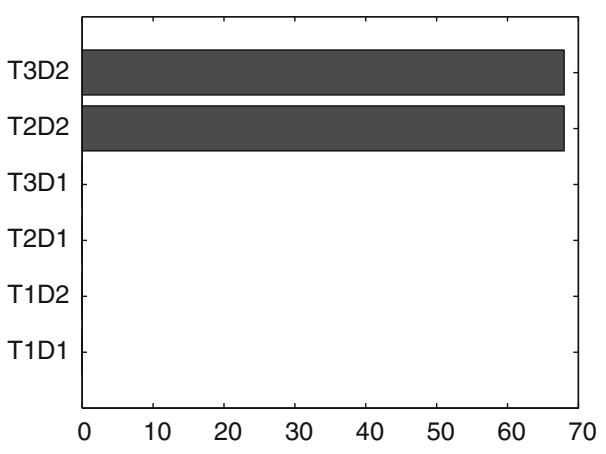

(e)

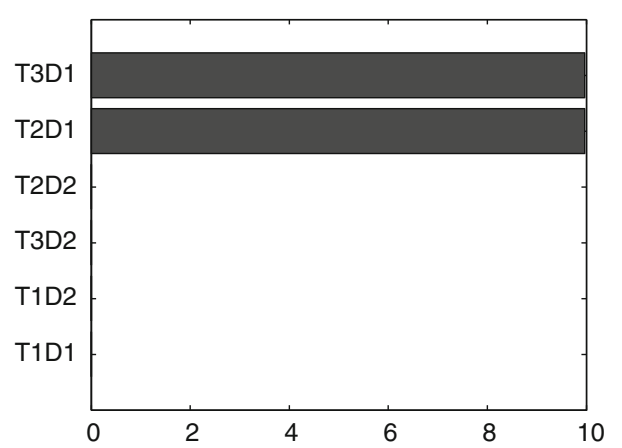

(b)

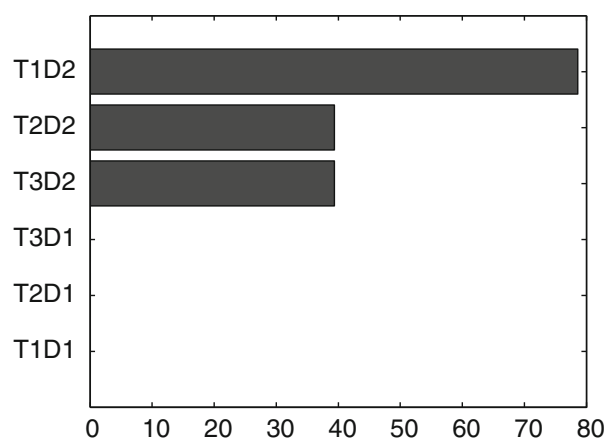

(d)

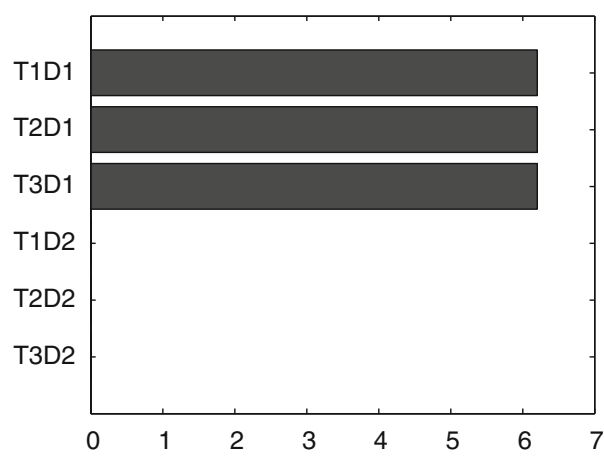

(f)
The scalability and symmetry of the discussed method deserve the following comments: (a) a scaled copy of the mechanism does not have the scaled I/O function of the original mechanism. A reason for this behavior is that size has effects on the flexibility of the material. (b) A scaled copy of the mechanism accepts the same DOCE methodology proposed, to reach its I/O function. (c) The DOCE method applied here does not make use of symmetries of the modeled mechanism. However, we did use symmetries in checking the values of the obtained coefficients (Eqs. 5, 6) with the purpose of finding possible modeling or calculation errors. Symmetries helped us to find and correct such procedural errors, reaching a meaningful equation.
Although it was not a goal for this article, future work might include the consideration of mechanism symmetries to find the coefficients (not only to check them).

\subsection{Validation of the HexFlex meta-model}

The resulting residuals patterns (i.e., error vs. predicted values) were found to be aleatory and normally distributed around zero leading to confirm that the error is additive. To validate the accuracy of the meta-model, 1,000 random experiments with uniform distributions and factor levels between -1 and $1 \mathrm{~N}$ are made. The resulting forward model is used to compare the estimations of the mechanism

\begin{tabular}{|c|c|c|c|}
\hline Journal : Large 366 & Dispatch : & $30-6-2012$ & Pages : 15 \\
\hline $\begin{array}{l}\text { Article No. : } \mathbf{2 6 8} \\
\text { MS Code : } \quad \text { EWCO-D-10-00078 }\end{array}$ & $\begin{array}{l}\square \\
\boldsymbol{V}_{\mathrm{CP}}^{\mathrm{LE}}\end{array}$ & & $\begin{array}{l}\square \text { TYPESET } \\
\boldsymbol{V} \text { DISK }\end{array}$ \\
\hline
\end{tabular}


Table 3 Lenth's analysis of six DOF HexFlex mechanism

\begin{tabular}{|c|c|c|c|c|c|c|}
\hline & $x(\mu \mathrm{m})$ & $y(\mu \mathrm{m})$ & $z(\mu \mathrm{m})$ & $\theta_{x}(\mu \mathrm{rad})$ & $\theta_{y}(\mu \mathrm{rad})$ & $\theta_{z}(\mu \mathrm{rad})$ \\
\hline T1D2 & 0.17 & 0 & 575,314 & 78.63 & 0.05 & 0 \\
\hline T2D2 & 0.17 & 0 & 575,317 & 39.36 & 68.07 & 0 \\
\hline T3D2 & 0.17 & 0 & 575,321 & 39.27 & 68.12 & 0 \\
\hline T2D1 & $57,527.50$ & $99,637.1$ & 0 & 0 & 0 & 6.20 \\
\hline T3D1 & $57,531.50$ & $99,635.9$ & 0 & 0 & 0 & 6.2 \\
\hline T1D1 & $115,053.50$ & 0 & 0 & 0 & 0 & 6.20 \\
\hline$v$ & 19.63 & 0 & 287,657 & 19.63 & 0.03 & 3.10 \\
\hline$S_{0}$ & 29.45 & 0 & 431,486 & 29.45 & 0.04 & 4.70 \\
\hline ME & 110.73 & 0 & 0 & 0 & 0 & 0 \\
\hline SME & 265.34 & 0 & 0 & 0 & 0 & 0 \\
\hline
\end{tabular}

Table 4 Uniform DOCE and results of the experiments

\begin{tabular}{|c|c|c|c|c|c|c|c|c|c|c|c|c|}
\hline \multicolumn{7}{|c|}{ Design matrix } & \multicolumn{6}{|c|}{ Responses } \\
\hline Treatment & $\begin{array}{l}\text { T1D1 } \\
\text { (N) }\end{array}$ & $\begin{array}{l}\text { T1D2 } \\
(\mathrm{N})\end{array}$ & $\begin{array}{l}\text { T2D1 } \\
(\mathrm{N})\end{array}$ & $\begin{array}{l}\text { T2D2 } \\
(\mathrm{N})\end{array}$ & $\begin{array}{l}\text { T3D1 } \\
(\mathrm{N})\end{array}$ & $\begin{array}{l}\text { T3D2 } \\
(\mathrm{N})\end{array}$ & $x(\mu \mathrm{m})$ & $y(\mu \mathrm{m})$ & $z(\mu \mathrm{m})$ & $\theta_{x}(\mu \mathrm{rad})$ & $\theta_{y}(\mu \mathrm{rad})$ & $\theta_{z}(\mu \mathrm{rad})$ \\
\hline 1 & 0.6 & -0.2 & -1.0 & -1.0 & 1.0 & -0.2 & -23.00 & 0.00 & -403.37 & $15,723.33$ & $27,294.40$ & $-8,057.19$ \\
\hline 2 & -0.6 & -0.6 & 0.2 & 1.0 & -1.0 & 0.2 & 0.00 & -39.85 & 172.88 & $-47,310.09$ & $-27,294.38$ & $5,583.32$ \\
\hline 3 & -0.2 & 0.2 & -0.2 & -1.0 & -1.0 & 0.6 & 11.5 & -59.78 & -57.63 & $15,769.99$ & $54,588.86$ & $3,098.05$ \\
\hline 4 & -1.0 & -0.2 & 1.0 & 0.6 & -0.2 & 1.0 & -23.01 & 39.85 & 403.37 & $-39,390.09$ & $13,647.29$ & $6,831.66$ \\
\hline 5 & 1.0 & 0.2 & 1.0 & 1.0 & 0.6 & 0.2 & 69.02 & 79.71 & 403.37 & $-15,723.33$ & $-27,294.40$ & $-1,883.93$ \\
\hline 6 & -1.0 & -1.0 & -0.2 & -1.0 & -0.2 & -1.0 & -57.52 & -19.93 & -864.35 & -116.71 & -0.06 & $3,120.86$ \\
\hline 7 & -0.6 & 0.2 & -1.0 & 1.0 & 0.2 & 0.6 & -69.02 & -39.85 & 518.61 & $-23,596.69$ & $-13,647.16$ & $-1,838.28$ \\
\hline 8 & 0.6 & -0.6 & 0.6 & 0.6 & 0.2 & -1.0 & 46.01 & 39.85 & -288.11 & $-15,816.67$ & $-54,588.89$ & -635.59 \\
\hline 9 & -1.0 & 1.0 & -0.6 & -0.2 & -0.6 & 0.2 & -57.52 & -59.78 & 288.10 & $39,483.43$ & $13,647.20$ & $3,120.87$ \\
\hline 10 & 0.2 & 1.0 & 0.2 & 1.0 & -0.2 & -0.6 & 23.01 & 0.00 & 403.35 & $31,610.14$ & $-54,588.88$ & 612.76 \\
\hline 11 & -0.6 & 0.6 & 0.2 & -1.0 & 0.2 & 1.0 & -34.51 & 19.93 & 172.86 & $23,690.01$ & $68,236.09$ & $1,872.51$ \\
\hline 12 & 1.0 & -1.0 & -0.2 & 0.6 & -0.6 & -0.2 & 69.02 & -39.85 & -172.86 & $-47,356.77$ & $-27,294.41$ & $-1,883.92$ \\
\hline 13 & -1.0 & 0.6 & -0.2 & 0.6 & 0.6 & -0.2 & -80.53 & 19.93 & 288.11 & $15,816.74$ & $-27,294.44$ & 646.99 \\
\hline 14 & -1.0 & 0.2 & 0.6 & -0.6 & 1.0 & -0.6 & -69.02 & 79.71 & -288.12 & $31,516.73$ & -0.05 & $1,883.92$ \\
\hline 15 & 1.0 & 1.0 & 0.6 & -1.0 & -0.2 & 0.2 & 80.52 & 19.93 & 57.61 & $55,230.10$ & $40,941.61$ & -646.99 \\
\hline 16 & 1.0 & 0.6 & -1.0 & 0.2 & 0.2 & -0.6 & 23.01 & -39.85 & 57.61 & $31,563.44$ & $-27,294.47$ & $-6,831.66$ \\
\hline 17 & 0.2 & 1.0 & -0.6 & -0.6 & 0.6 & -1.0 & -23.01 & 0.00 & -172.89 & $70,976.84$ & $-13,647.30$ & $-4,334.97$ \\
\hline 18 & 0.6 & 0.6 & -0.6 & 0.6 & -1.0 & 1.0 & 46.02 & -79.71 & 633.86 & $-7,803.34$ & $13,647.27$ & -635.57 \\
\hline 19 & 0.2 & 0.2 & 0.6 & 0.2 & -0.6 & -0.2 & 46.01 & 0.00 & 57.62 & $7,896.70$ & $-13,647.22$ & $3,086.63$ \\
\hline 20 & 0.6 & -0.2 & 0.2 & -0.6 & -1.0 & -0.6 & 69.02 & -39.85 & -403.37 & $15,723.35$ & -0.04 & $1,838.29$ \\
\hline 21 & 0.6 & -0.6 & 1.0 & -0.6 & -0.6 & 0.6 & 80.52 & 19.93 & -172.86 & $-23,690.10$ & $40,941.67$ & $3,075.22$ \\
\hline 22 & 0.2 & -1.0 & 0.2 & 0.2 & 1.0 & 1.0 & -11.50 & 59.78 & 57.64 & $-63,103.51$ & $27,294.51$ & $-3,098.05$ \\
\hline 23 & -0.2 & 0.6 & 1.0 & -0.2 & -1.0 & -1.0 & 46.01 & 0.00 & -172.88 & $47,310.13$ & $-27,294.50$ & $6,808.84$ \\
\hline 24 & -0.6 & -1.0 & 1.0 & -0.2 & 0.6 & -0.2 & -23.01 & 79.71 & -403.35 & $-31,610.10$ & 0.00 & $3,109.44$ \\
\hline 25 & -0.2 & -0.6 & -0.6 & -0.6 & 0.2 & 0.2 & -34.51 & -19.93 & -288.11 & $-15,816.74$ & $27,294.44$ & $-1,849.69$ \\
\hline 26 & -0.2 & -0.6 & -0.6 & 1.0 & 1.0 & -0.6 & -57.52 & 19.93 & -57.62 & $-31,563.37$ & $-54,588.85$ & $-4,323.57$ \\
\hline 27 & -0.6 & -0.2 & -1.0 & 0.2 & -0.6 & -1.0 & -46.01 & -79.71 & -288.12 & $7,850.04$ & $-40,941.69$ & 635.59 \\
\hline 28 & -0.2 & 1.0 & 0.6 & 0.2 & 1.0 & 0.6 & -23.01 & 79.71 & 518.60 & $23,736.74$ & $13,647.23$ & -612.77 \\
\hline 29 & 1.0 & -0.2 & -0.2 & -0.2 & 0.6 & 1.0 & 34.51 & 19.93 & 172.88 & $-23,643.42$ & $40,941.70$ & $-5,594.73$ \\
\hline 30 & 0.2 & -1.0 & -1.0 & -0.2 & -0.2 & 0.6 & -11.50 & -59.78 & -172.85 & $-47,356.81$ & $27,294.48$ & $-3,098.03$ \\
\hline
\end{tabular}

\begin{tabular}{|l|lll|}
\hline & Journal : Large 366 & Dispatch : 30-6-2012 & Pages : 15 \\
Article No. : $\mathbf{2 6 8}$ & $\square$ LE & $\square$ TYPESET \\
MS Code : EWCO-D-10-00078 & $\checkmark_{\text {CP }}$ & $\checkmark$ DISK \\
\hline
\end{tabular}


configuration using meta-modeling against the FEA software ANSYS $^{\odot}$. The precision of the model is calculated using three error criteria:

1. The maximum absolute error (MAXABS Eq. (7)).

2. The relative error between the meta-model and the FEA model.

3. The root mean square error (RMSE Eq. (8)) over the set of experiments.

The MAXABS and relative $\%$ of error allow to calculate the local error. The RMSE provides good estimate of the global error. The error between meta-model predictions and ANSYS $^{\odot}$ results is shown in Table 5. The deformed shape of the mechanism for one of the experiments made to validate the accuracy of the meta-model is in Fig. $3 \mathrm{f}$.

MAXABS $=\max \left\{\left|\psi_{i}-\widehat{\psi_{i}}\right|\right\}_{i=1, \ldots, n_{\text {error }}}$

$\operatorname{RMSE}=\sqrt{\frac{\sum_{i=1}^{n_{\text {error }}}\left(\psi_{i}-\widehat{\psi}_{i}\right)^{2}}{n_{\text {error }}}}$

where $\psi_{i}$ refers to $x, y$, and $z$ or the corresponding angles $\theta_{x}, \theta_{y}$, and $\theta_{z}$ as shown in Table 5. The maximum linear absolute error is in the $y$ direction $\left(4.18 \times 10^{-4} \mu \mathrm{m}\right)$. We compare this error with an ISO h6 manufacturing tolerance calculated on a shaft of nominal diameter $50 \mathrm{~mm}(50 \mathrm{~mm}$ $\left.{ }_{-19}^{+0} \mu \mathrm{m}\right)$ finding the error as acceptable. The maximum relative error is in the $y$ direction $(0.621 \%)$. This represents a better accuracy than that reported in Ref. [45] where the HexFlex is analyzed using a virtual method based on Euler beam theory and the results are also compared with a FEA software, obtaining a maximum relative error of $3 \%$.

\section{Discussion}

\subsection{Applicability to other mechanisms}

Design of experiments, and DOCE, in particular, are methods that can be applied to complex systems whose I/O function is fully or partially unknown. A mechanism is not

Table 5 Error between meta-model estimations and ANSYS ${ }^{\odot}$ simulations for 1,000 random experiments with uniform distribution

\begin{tabular}{llll}
\hline & MAXABS & MAX (\%error) & RMSE \\
\hline$x(\mu \mathrm{m})$ & $4.01 \times 10^{-4}$ & $1.08 \times 10^{-3}$ & $8.67 \times 10^{-5}$ \\
$y(\mu \mathrm{m})$ & $4.18 \times 10^{-4}$ & $3.59 \times 10^{-4}$ & $2.07 \times 10^{-5}$ \\
$z(\mu \mathrm{m})$ & $2.85 \times 10^{-4}$ & $2.41 \times 10^{-4}$ & $4.19 \times 10^{-5}$ \\
$\theta_{x}(\mu \mathrm{rad})$ & $2.41 \times 10^{-2}$ & $2.26 \times 10^{-3}$ & $9.78 \times 10^{-4}$ \\
$\theta_{y}(\mu \mathrm{rad})$ & 4.16 & $6.21 \times 10^{-1}$ & $5.57 \times 10^{-3}$ \\
$\theta_{z}(\mu \mathrm{rad})$ & $2.10 \times 10^{-2}$ & $2.30 \times 10^{-3}$ & $2.23 \times 10^{-3}$ \\
\hline
\end{tabular}

different, in this sense, to a chemical or a biotechnological process. DOCE is able to model other mechanisms, even if they are not of the HexFlex types. The numerical models obtained for different mechanisms will indeed be different in the classification of input and output variables, sensitivities, etc. However, the DOCE will still be valid in finding the I/O function of such mechanisms.

\subsection{Future work}

The use of DOCE to yield a transfer function for real time control of CMs has been addressed without considering non-ideal conditions relevant to actual working conditions of the mechanism. We could number a few of these nonidealities: (1) deformation due to wear which would lead to significant changes in dimensions, (2) shape variations due to temperature changes and (3) hysteresis, or material memory. We would expect the initially obtained transfer function by DOCE to be adjusted during the operation of the mechanism but we do not envision using neural networks to obtain CMs transfer functions because DOCE is more efficient and less time consuming.

Due to the symmetrical shape of the HexFlex CM and that it functions at a very narrow vicinity around the operation point, the resulting low-order polynomial linear meta-models obtained seem realistic and for that reason we consider that it should be interesting to test other compliant mechanisms with no symmetry to see whether linear regression models are adequate or not statistically and to consider using Kriging meta-models for larger experimental areas [39]. In addition, it would also be worthwhile to model well-known mechanisms (not necessarily CMs) to verify that their already known transfer functions also result from DOCE.

\section{Conclusions}

This article presents a computer-based meta-model for force-displacement transfer function of CMs under quasistatic conditions using DOCE. A case study is discussed in the domain of a six degrees of freedom HexFlex CM. To obtain the meta-model of the HexFlex, computer experiments based on Plackett-Burman and uniform DOCE are performed using FEA. The obtained meta-model of the HexFlex is linear for the movement range of the mechanism. The accuracy of the meta-model was calculated by running 1,000 FEA-based computer experiments. The values found in the experiment were compared against those generated by the meta-model. The results of the comparison can be observed in Table 5. They allow to conclude that the chosen meta-model is consistent around the operating point. 
The factorial DOCE permitted to identify/confirm characteristics of the mechanism, such as the presence of symmetries in the actuation and the quasi-static behavior of the mechanism. A uniform DOCE was employed to fine tune the model of the mechanism. The mechanism was modeled using a low-order polynomial, because of its quasi-static behavior and small displacements. The resulting I/O model of the mechanism allows having a transfer function for developing real-time control. It should be noted that the (linear) model obtained is easily invertible, which adds to its applicability. However even if the model obtained was not linear, its invertibility is guaranteed because it is a polynomial approximation around the vicinity of the operating point.

Nonetheless, DOCE is not intended to replace real experiment-based DOE but to forecast/ignore possible interactions and to fine-tune ranges, therefore, reducing costs of experimentation and model/product development.

Acknowledgments Support for this work was provided by the Colombian Administrative Department of Sciences, Technology and Innovation (COLCIENCIAS) and the Colombian National Service of Learning (SENA) grant No. 1216-479-22001. The authors gratefully acknowledge this support.

\section{References}

1. Luo Z, Tong L, Wang M, Wang S (2007) Shape and topology optimization of compliant mechanisms using a parameterization level set method. J Comput Phys 227(1):680-705

2. Jensen B, Howell L (2002) The modeling of cross-axis flexural pivots. Mech Mach Theory 37(5):461-476

3. Howell L (2001) Compliant mechanisms. Wiley, New York

4. Lan C, Lee K (2006) Generalized shooting method for analyzing compliant mechanisms with curved members. J Mech Design 128(4):765-775

5. Venanzi S, Giesen P, Parenti-Castelli V (2005) A novel technique for position analysis of planar compliant mechanisms. Mech Mach Theory 40(11):1224-1239

6. Booker A, Dennis JE, Frank P, Serafini D, Torczon V, Trosset W (1997) Optimization using surrogate objectives on a helicopter test example. In: Computational methods in optimal design and control. Birkhäuser, Boston, pp 49-58

7. Gu L (2001) A comparison of polynomial based regression models in vehicle safety analysis. In: ASME 2001 design engineering technical conferences. DETC2001, Pittsburgh

8. Simpson T, Booker A, Ghosh D, Giunta A, Koch P, Yang R (2004) Approximation methods in multidisciplinary analysis and optimization: a panel discussion. Struct Multidiscip O 27(5): 302-313

9. Kodiyalam S, Yang R, LEI G (2004) High-performance computing and surrogate modeling for rapid visualization with multidisciplinary optimization. AIAA J 42(11):2347-2354

10. Simpson T, Poplinski J, Koch P, Allen J (2001) Metamodels for computer-based engineering design: survey and recommendations. Eng Comput 17(2):129-150

11. Crary S (2002) Design of computer experiments for metamodel generation. Analog Integr Circ S 32(1):7-16
12. Culpepper M, Anderson G, Petri P (2002) Hexflex: A planar mechanism for six-axis manipulation and alignment. In: ASPE Annual Meeting, ASPE

13. Culpepper M (2007) Multiple degree of freedom compliant mechanism. United States Patent, Number: US007270319B

14. Kim C, Moon Y, Kota S (2008) A building block approach to the conceptual synthesis of compliant mechanisms utilizing compliance and stiffness ellipsoids. J Mech Design 130(2):2308-1-11

15. Morsch F, Tolou N, Herder J (2009) Comparison of methods for large deflection analysis of a cantilever beam under free end point load cases. In: ASME 2009 design engineering technical conferences. DETC2009, San Diego

16. Trease B, Moon Y, Kota S (2005) Design of large-displacement compliant joints. J Mech Design 127(4):788-798

17. Dibiaso C, Culpepper M, Panas R, Howell L, Magleby S (2008) Comparison of molecular simulation and pseudo-rigid-body model predictions for a carbon nanotube based compliant parallel-guiding mechanism. J Mech Design 130(04):2302-1-8

18. Chang S, Lee J, Yen H (2005) Kinematic and compliance analysis for tendon-driven robotic mechanisms with flexible tendons. Mech Mach Theory 40(6):728-739

19. Yong Y, Lu T (2009) Kinetostatic modeling of 3-RRR compliant micro-motion stages with flexure hinges. Mech Mach Theory 44(6):1156-1175

20. Lobontiu N, Paine J, Garcia E, Goldfarb M (2002) Design of symmetric conic-section flexure hinges based on closed-form compliance equations. Mech Mach Theory 37(5):477-498

21. Lobontiu N, Paine J, Garcia E, Goldfarb M (2001) Corner-filleted flexure hinges. J Mech Design 123(3):346-352

22. Dado M (2005) Limit position synthesis and analysis of compliant 4-bar mechanisms with specified energy levels using variable parametric pseudo-rigid-body model. Mech Mach Theory 40(8):977-992

23. Park SR, Yang ST (2005) A mathematical approach for analyzing ultra precision positioning system with compliant mechanism. J Mater Process Tech 164-165:1584-1589

24. Banerjee A, Bhattacharya B, Mallik A (2009) Forward and inverse analyses of smart compliant mechanisms for path generation. Mech Mach Theory 44(2):369-381

25. Boyle C, Howell L, Magleby S, Evans M (2003) Dynamic modeling of compliant constant-force compression mechanisms. Mech Mach Theory 38(12):1469-1487

26. Tolou N, Herder J (2009) A seminalytical approach to large deflections in compliant beams under point load. Math Probl Eng 2009:13

27. Zettl B, Szyszkowski W, Zhang W (2005) Accurate low DOF modeling of a planar compliant mechanism with flexure hinges: the equivalent beam methodology. Precis Eng 29(2):237-245

28. DeBona F, Munteanu M (2005) Optimized flexural hinges for compliant micromechanisms. Analog Integr Circ S 44(2): 163-174

29. Saxena A, Ananthasuresh G (2001) Topology synthesis of compliant mechanisms for nonlinear force-deflection and curved path specifications. J Mech Design 123(1):33-42

30. Zhang S, Fasse E (2001) A finite-element-based method to determine the spatial stiffness properties of a notch hinge. J Mech Design 123(1):141-147

31. Rao CR (1967) Least squares theory using an estimated dispersion matrix and its application to measurement of signals. In: 5Th Berkeley symposium on mathematical statistics and probability, vol I. University of California Press, Berkeley, pp 355-372

32. Plackett RL, Burman JP (1946) The design of optimal multifactorial experiments. Biometrika 33 (4):305-325

33. Fang K, Li R (2006) Uniform design for computer experiments and its optimal properties. Int J Mater Prod Tec 25(1-3):198-210

$\begin{array}{lll}\text { Journal : Large } \mathbf{3 6 6} & \text { Dispatch : 30-6-2012 } & \text { Pages : } \mathbf{1 5} \\ \text { Article No. : } \mathbf{2 6 8} & \square \text { LE } & \square \text { TYPESET } \\ \text { MS Code : EWCO-D-10-00078 } & \boldsymbol{V}_{\mathrm{CP}} & \checkmark \text { DISK }\end{array}$


34. Culpepper M, Anderson G (2004) Design of a low-cost nanomanipulator which utilizes a monolithic, spatial compliant mechanism. Precis Eng 28(4):469-482

35. Craig J (1989) Introduction to Robotics, 2nd edn. Addison Wesley, USA

36. Paul R (1981) Robot manipulators: mathematics, programming, and control. MIT Press, Cambridge

37. Box G, Hunter J, Hunter W (2005) Statistics for experimenters: design, innovation, and discovery, 2nd edn. Wiley, New York

38. NIST (2008) Engineering statistics handbook.nist/sematech e-handbook of statistical methods

39. Kleijnen J (2008) Design and analysis of simulation experiments. Springer, Heidelberg

40. Lenth RV (1989) Quick and easy analysis of unreplicated factorials. Technometrics 31(4):469-473
41. Fang KT, Lin DK, Winker P, Zhang Y (2000) Uniform design: theory and application. Technometrics 42(3):237-248

42. Simpson TW, Peplinski J, Koch PN, Allen JK (1997) On the use of statistics in design and the implications for deterministic computer experiments. In: ASME 1997 design engineering technical conferences, DETC1997

43. Bates DM, DebRoy S (2004) Linear mixed models and penalized least squares. J Multivariate Anal 91(1):1-17

44. Ruppert D, Wand MP (1994) Multivariate locally weighted least squares regression. Ann Stat 22(3):1346-1370

45. Petri $P$ (2002) A continuum mechanic design aid for non-planar compliant mechanisms. M.Sc thesis, MIT press Cambridge 